technology of substances of critical importance to the project. However, in the case of heavy water and graphite production and the reduction of uranium compounds to metal, it has been agreed that some of the older production processes can now be declassified. All information on the neutron crosssections of carbon and graphite has been declassified. The spontaneous fission-rate of plutonium-240 has been declassified. As a result of previous conferences, values for the thermal neutron cross-sections of uranium-235, uranium-238, natural uranium and plutonium-239 were released. It has now been agreed that the cross-sections may be published in the ordinary way, together with experimental details of the method of measurement. Much of the basic chemistry of plutonium has already been declassified. It has now been agreed that some of the physical properties that are related to the metallurgy of pure plutonium can also be released. Basic chemical studies involving the use of ion exchange resins for all elements except plutonium can be released, as can basic studies on the chemical kinetics of fluoride corrosion. Information can now be released on the production of fission products as sources of radiation for industrial and medical purposes.

\section{Scientific Requirements of Pakistan}

Ar the Sixth Pakistan Science Conference, held in Karachi during January 18-23, the presidential address was given by Dr. M. Raziuddin Siddiqi, vice-chancellor of the University of Peshawar, who chose as his subject "Scientific Research in the East". After referring to the fact that the early development of all sciences owed much to the scholars and savants of the East, who at the dawn of history were both leaders and sole workers in all branches of knowledge, Dr. Siddiqi affirmed that freedom of inquiry and freedom of expression are the two essential conditions for any kind of intellectual activity. Learning and scholarship thrive, he said, only where and when the true democratic spirit prevails, and the neglect of these conditions has been an important factor in the decay of scientific activity in the East during the past few centuries. Another factor has been the distortion of values and lack of appreciation of intellectual achievement : even now it is not generally recognized that education and research are as essential elements in national defence as armed forces, and that scientific education and research have their place in any long-range defence plan.

The immediate problem for Pakistan, Dr. Siddiqi continued, is not so much that of providing mechanics, technicians or science teachers as to produce the experts and research workers of the highest standard who can discover and develop its natural resources, build up new industries, and design and instal the plant required. An intensive search for talent among students is required and, when found, the prospective experts and investigators should be given a much longer training than is at present customary. Status and remuneration also require attention, and good work in the scientific and technical field should be sure of ample recognition. Adequate provision should also be made for fundamental research, and this is a matter of equipment as well as of personnel. Apart from strengthening the research work at the universities, well-planned and equipped scientific academies and research instifutes are required, and it is also desirable to remedy the failure of the existing organ. ization for research to produce the apparatus and equipment essential for research purposes. Dr. Siddiqi emphasized that Pakistan cannot always rely on other countries for the supply of the essential tools of research.

\section{Plankton Studies}

Ir is a matter of common observation that plankton is very unevenly distributed in the sea and, moreover, frequently it appears to be the case that any one patch may consist almost entirely of phytoplankton while another is mainly zooplankton. Two principal theories, not mutually exclusive, have been put forward to account for this. One, associated with the name of Harvey, is that the zooplankton would graze down a patch of phytoplankton, increasing correspondingly in numbers as it does so, until the phytoplankton is used up, and this would be followed by the dying off or migration of the zooplankton, leaving a more or less free area in which the phytoplankton could once again forge ahead. The other, associated with the name of Hardy, suggests that a high concentration of phytoplankton in some way actually repels the zooplankton and keeps it from invading the area. Richard Bainbridge (J.Mar. Biol. Assoc., 32, 385; 1953) has approached the whole problem from the experimental angle. Several ingenious pieces of apparatus made of rectangular 'Perspex' tubes, including a circular channel $4 \mathrm{ft}$. in diameter with an internal diameter $2 \times 1 \frac{1}{2}$ in., made it possible to follow the migration of plankton, horizontally and vertically, under controlled conditions. An arrangement of sliding water-tight doors and admission points allowed of the setting up of phytoplankton gradients and the subsequent introduction of zooplankton. On the whole, using a fairly wide range of organisms, the evidence obtained favours the 'grazing' hypothesis, but with active and directive migration, both horizontal and vertical, playing a larger part than perhaps had been envisaged previously. In initial experiments it was found that none of the diatoms used, with the possible exception of Nitzschia in very high concentration, showed a toxic or excluding effect.

\section{Activity of Fungi in Dried Soil}

Is relation to the very considerable work now in progress both in the fields of antibiotics and plant pathology, it is essential that fungal cultures can be stored for considerable periods of time without losing their pristine physiological properties; in particular, their pathogenicity and their ability to produce particular substances under specified conditions. In a recent paper, R. G. Atkinson (Canad. $J$. Bot., 31, 5, 542; 1953) has reviewed some of the methods which have been used for storing fungal cultures and has contributed new data on the survival and pathogenicity of Alternaria raphani after five years in dried soil culture. Soil cultures of Alternaria raphani, a seed-borne pathogen of radish, that had become dry when four to six weeks old, were still viable after five years. The incidence of residual viable centres in cultures of four of the five wild types studied was much higher than in those of their respective variants, on the basis of colony counts from platings of 5 -gm. samples diluted onefifth. The reverse of this was true for the remaining wild type and its variant. This pair was also unique in that the wild type had been shown to possess little infectivity, whereas its variant had proved to be highly virulent. The fungus showed no change in cultural characteristics after its five-year 